\title{
The effectiveness of implementing a reminder system into routine clinical practice: does it increase postpartum screening in women with gestational diabetes?
}

\author{
A. K. Shea, PhD, MSc (1); B. R. Shah, MD, PhD (2,3,4); H. D. Clark, MD, MSc (5,7); J. Malcolm, MD (5); \\ M. Walker, MD, MSc (6,7); A. Karovitch, MD, MEd (5,6); E. J. Keely, MD $(5,6)$
}

\begin{abstract}
Introduction: During regular care, women with previous gestational diabetes mellitus (GDM) rarely receive the recommended screening test for type 2 diabetes, a 2-hour oral glucose tolerance test (OGTT), in the postpartum period. The current study examined whether the implementation of a reminder system improved screening rates.
\end{abstract}

Methods: Based on our previous randomized control trial, we implemented a postpartum reminder (letter or phone call) protocol into routine care at two of three clinical sites. We verified postpartum testing by searching hospital laboratory databases and by linking to the provincial physician service claims database. The primary outcome was the proportion of patients who underwent an OGTT within 6 months of delivery.

Results: Women who received care in a setting using a reminder system were more likely to receive an OGTT within 6 months postpartum (28\%) compared with usual care (14\%). The OGTT rates for both reminder groups were lower than that found in our randomized control trial ( $28 \%$ vs. $60 \%)$.

Conclusion: Although the screening rates remain low, postpartum reminders doubled screening rates using the recommended test, the OGTT.

Keywords: gestational diabetes, postpartum, screening, reminders,

type 2 diabetes prevention

\section{Introduction}

Gestational diabetes mellitus (GDM), defined as hyperglycemia at the onset of pregnancy or first recognized in pregnancy, affects about $3 \%$ to $4 \%$ of nonAboriginal women and up to $18 \%$ of Aboriginal women in Canada. ${ }^{1,2}$ Although GDM and gestational impaired glucose tolerance (IGT) are associated with poor obstetrical outcomes, the most serious public health concern may be the 7 -fold increased risk of developing type 2 diabetes (T2DM) compared to women with normal glucose tolerance in pregnancy. ${ }^{3-6}$ The Canadian Diabetes Association (CDA) recommends that women with GDM have a 2-hour oral glucose tolerance test (OGTT) at 6 weeks to 6 months postpartum.? Recommendations from the International Workshop Conference on GDM suggest screening at 6 weeks postpartum using the 75-gram, 2-hour OGTT, which should then be repeated at one-year postpartum and then at least every 3 years thereafter. ${ }^{8}$ A fasting glucose blood test alone misses approximately $40 \%$ of those with diabetes and fails to identify those with IGT. ${ }^{9}$ When screened between 6 weeks and 3 months postpartum, $13 \%$ to $32 \%$ of women with GDM have IGT that may persist or later develop into T2DM. ${ }^{10,11}$ The postpartum period therefore presents a unique opportunity for the identification of women at high risk of developing diabetes and provides an important opportunity for early intervention and prevention.

Although the importance of postpartum screening with an OGTT is known, screening rates remain disappointingly low in routine clinical practice. ${ }^{12-14}$ Identified barriers to implementing the recommended postpartum screenings include poor communication between obstetrician and primary care provider, providers uncertain about screening recommendations, patients unaware of the risk of not screening and patients missing screening appointments due to competing time commitments. ${ }^{15}$ Our group previously identified that the majority of women were not receiving the recommended postpartum screening in the Ottawa area of Ontario, Canada. ${ }^{12}$ We did a randomized control trial (RCT) at The Ottawa Hospital (TOH) where the woman, her family physician, both of them or neither of them received a postal reminder at approximately 3 months postpartum to have an OGTT completed. If either the woman, the physician or both received the reminder, screening rates increased 4-fold from $14 \%$ (no reminder) to approximately $60 \%$. Approximately $30 \%$ of the women in that study who completed the OGTT had an abnormal result. ${ }^{16}$

Author references:

1. Faculty of Medicine, University of Ottawa, Ottawa, Ontario, Canada

2. Institute for Clinical Evaluative Sciences, Toronto, Ontario, Canada

3. Department of Medicine, University of Toronto, Toronto, Ontario, Canada

4. Department of Medicine, Sunnybrook Health Sciences Centre, Toronto, Ontario, Canada

5. Department of Medicine, The Ottawa Hospital and University of Ottawa, Ottawa, Ontario, Canada

6. Department of Obstetrics \& Gynecology, The Ottawa Hospital and University of Ottawa, Ottawa, Ontario, Canada

7. Ottawa Hospital Research Institute, Clinical Epidemiology Program, The Ottawa Hospital and University of Ottawa, Ottawa, Ontario, Canada

Correspondence: Dr. Erin Keely, Division of Endocrinology and Metabolism, The Ottawa Hospital, 4th Floor Riverside Campus, 1967 Riverside Drive, Ottawa, Ontario, Canada K1H 7W9;

Tel.: (613) 738-8400 Ext. 81946; Fax: (613) 737-8396; Email: ekeely@ottawahospital.on.ca 
Based on the results from the RCT, we implemented a reminder system into routine practice in two of three GDM clinical sites in the Ottawa area. The third site could not institute the reminder system due to logistics. The aim of our current study was to compare whether our implementation of the reminder system at the two sites made a difference in screening rates using the CDA-recommended 2-hour OGTT. We hypothesized that the women who had received care at sites implementing the reminder system would have a higher postpartum screening rate using an OGTT compared to those who had not. The analyses were based on an intention-to-treat model.

\section{Methods}

This study was approved by the Research Ethics Committee of both The Ottawa Hospital ( $\mathrm{TOH}$ ) and the Queensway Carleton Hospital (QCH). TOH is a university-affiliated tertiary centre in Ottawa, Ontario, Canada, that provides services to a catchment area of 900000 people. TOH provides obstetrical services at two of its campuses, the Civic Campus (west) and the General Campus (east), each of which performs approximately 3500 deliveries per year. The QCH is a community-based hospital in the west end of the city that serves a population of over 400000 and provides 2800 deliveries per year. Most women in the region are referred to one of these three sites for management of their GDM. All primary care is provided by family physicians.

We identified all the attendees at GDM education classes at one of these three sites between July 1, 2007, and June 30, 2008. Women were diagnosed with GDM following either a 50-g glucose challenge test (GCT) or a 75-g OGTT using the CDA practice guidelines criteria. ${ }^{7}$ A GDM diagnosis was made if the woman had either (1) a 50-g GCT with a plasma glucose level greater than or equal to $10.3 \mathrm{mmol} / \mathrm{L}$ or (2) a 75-g OGTT with two of the following three results: fasting plasma glucose level greater than or equal to $5.3 \mathrm{mmol} / \mathrm{L}$; 60-minute plasma glucose levels greater than or equal to $10.6 \mathrm{mmol} / \mathrm{L}$; or 120-minute plasma glucose level greater than or equal to $8.9 \mathrm{mmol} / \mathrm{L}^{7}$ All the GDM education classes give information on the risks of GDM including the development of diabetes postpartum, on individualized nutrition counselling and on monitoring blood glucose at home, and patients return to the site for ongoing GDM care. At reminder site A ( $\mathrm{TOH}$, General Campus) and $\mathrm{B}(\mathrm{QCH})$ women were seen by an endocrinologist/internist for ongoing care and continued with their usual obstetrical care provider; at the non-reminder site (TOH, Civic Campus), women were seen by a high-risk obstetrician and obstetrical care could be transferred to the site if need be. Patients were referred to an internist if assistance with insulin management was required. Similar protocols for insulin initiation, based on the CDA recommendations, were used at all sites.

For Ontario residents, all physician visits, medical care and diagnostic testing are covered by the Ontario Health Insurance Plan (OHIP), provincial health insurance that is universally available without copayment. If patients lacked OHIP coverage, they were excluded from the study since postpartum testing could not otherwise be verified.

Approximately three months after delivery, patients from reminder site A were mailed a reminder that included information on the importance of diabetes screening and a laboratory requisition for an OGTT at a non-hospital-based laboratory, and patients from reminder site B were either sent a letter with a laboratory requisition or phoned or both. Patients from the non-reminder site did not receive a postal reminder or a reminder phone call. No sites provided routine postpartum follow-up visits for GDM.

We collected baseline characteristics and obstetrical outcomes by reviewing patient charts. We estimated socio-economic status using the neighbourhood income quintile, according to the patient's home postal code. Ethnicity and education level were not available. We searched through two sources to identify diabetes screening tests for the study participants: the hospitalbased electronic record system to identify whether participants had completed postpartum diabetes screening at the hospital laboratory and records of billing claims from community laboratories, by linking each participant to the provincial physician service claims database using their unique health care number through the Institute for Clinical Evaluation Services (ICES). Because of the single-payer universal health care system in Ontario, the database includes information on all laboratory testing performed outside of the hospital setting, including the types and dates of tests. (The results of the laboratory tests are not available from these databases.)

The primary outcome was the proportion of patients who were screened for T2DM with an OGTT within 6 months of delivery. Two additional outcomes were (1) an alternate test that might have be used to screen for T2DM within 6 months of delivery (random glucose test, fasting glucose test or glycated hemoglobin [HbA1c] test) or (2) any test that might be used to screen for T2DM within 6 months of delivery (OGTT, random glucose test, fasting plasma glucose (FPG) test or HbA1c test).

Using chi-square tests and analysis of variance (ANOVA), we compared the baseline demographic, clinical and metabolic parameters between study participants and excluded participants and between the three sites. To compare screening rates at the three sites, we performed a chi-square test for each outcome versus site of delivery. We used logistic regression analyses to adjust for the following baseline characteristics: maternal age at delivery; socio-economic status; previous GDM; pre-pregnancy body mass index (BMI; normal vs. overweight vs. obese); GDM treatment (diet only vs. insulin); and family history of T2DM. We report unadjusted means and associated standard deviations for continuous variables, as well as the proportion of participants for each variable of interest (number and percent) for categorical variables. Differences are considered significant at $p \leq .05$.

\section{Results}

We identified a total of 349 cases who attended the education classes at the three sites. Of these, 60 women did not meet the criteria for GDM and 27 did not have OHIP coverage (mostly because they were residents of the neighbouring province of Quebec) leaving 262 participants. The 27 excluded women differed from the 
included participants in that there was a significantly greater proportion of cigarette smokers in the excluded group ( $p=.002$ ); they also gave birth to infants with a lower weight ( $p=.028$ ). However, the difference in birth weight did not remain significant when gestational age at delivery was controlled for $(p=.316)$. There were no other significant differences compared to the included participants, as seen in Table 1.

The majority of women $(96.6 \% ; 253 / 262)$ returned to the site for GDM care following their education class. Of the participants from the reminder sites, $92.2 \%(83 / 90)$ returned to reminder site A and $100 \%$ $(55 / 55)$ returned to reminder site $B$, and were followed by an internist/endocrinologist for GDM care. Of the participants from the non-reminder site, $98.3 \%(115 / 117)$ returned and all but three were seen by the high risk obstetrician for GDM care; of these, $17.9 \%(21 / 117)$ were also seen by an internist/endocrinologist.

Hospital birth records were available for $91.6 \%(240 / 262)$ of participants. There were no differences among the sites for the following characteristics: birth weight; proportion of infants born > $4000 \mathrm{~g}$; proportion of Caesarian sections; proportion of multiple gestation; proportion of primigravids or proportion of women experiencing preeclampsia (See Table 1). Women from the non-reminder site, however, did give birth earlier than women from reminder site $B$ (38 vs. 39 weeks, $p=.010$ ).

At reminder site A, $96.7 \%$ of participants were sent a postal reminder with a laboratory requisition ( $\mathrm{n}=3$ not sent) following delivery, but $11.5 \%(10 / 87)$ of these were returned because the patient no longer lived at that address. Of the participants from reminder site B, $76.3 \%(42 / 55)$ were mailed a reminder letter, $14.5 \%(8 / 55)$ were phoned by a volunteer, and $7.3 \%$ $(4 / 55)$ received both a letter and a phone call; one could not be reached by telephone and no letter was sent. No patients from the non-reminder site received postal reminders or phone calls.

In the intention-to-treat analysis using all participants, $21.7 \%$ (57/262) women completed the OGTT postpartum screening within 6 months of delivery, $23.3 \%(21 / 90)$ from reminder site A, $36.4 \%(20 / 55)$ from reminder site B and $13.7 \%$ (16/117) from the non-reminder site ( $p=.01$ ) (Table 2). When the reminder sites are combined, $28 \%(41 / 145)$ completed the OGTT, significantly more than those from the nonreminder site (chi-square $\left[\chi^{2}\right]=7.274$; $p=.01$; degrees of freedom $[\mathrm{df}]=1$ ) . In the logistic regression analyses, significantly more women from reminder site B completed an OGTT compared to the non-reminder site (adjusted odds ratio $[\mathrm{OR}]=3.10 ; p=.03$ ); reminder site $\mathrm{A}$ did not differ from either site in OGTT completion (Table 3). When we examined the occurrence of any glucose test (random/ fasting glucose test, HbA1c or OGTT) in the 6 months following delivery, we found that $41.6 \%(109 / 262)$ of women had completed one or more of these tests. Of the 57 women who had had OGTTs, $81 \%$ ( $n=46$ ) had records of the test in the community laboratory billing claims database. There were no statistically significant differences among the sites for the proportion of women who completed either random/ fasting glucose testing or HbA1c or any diabetes screening test.

Factors that may influence screening rates were entered into a logistic regression analysis (Table 3). Women treated using diet only (vs. insulin) were less likely to complete the OGTT (adjusted OR $=0.38$; $\mathrm{CI}=$ $0.18-0.80 ; p=.01$ ). No other factors were found as significant predictors of OGTT testing. Paradoxically, women who were normal weight or overweight (vs. obese) were more likely to go for any postpartum glucose test (adjusted $\mathrm{OR}=2.40 ; \mathrm{CI}=$ 1.16-5.01 and adjusted $\mathrm{OR}=3.10 ; \mathrm{CI}=$ 1.42-6.77, respectively; $p=.03$ ). A family history of T2DM and previous GDM did not have a significant effect on the participant undergoing postpartum diabetes screening.

\section{Discussion}

We found that women with previous GDM who received care at sites where reminders are used for postpartum diabetes screening were more likely to receive the recommended test, the OGTT. The OGTT rates for both reminder groups were lower than that found in our RCT ( $28 \%$ vs. $60 \%$ ), as expected in a comparative effectiveness study. Our rates of screening without reminders had not improved $(13.7 \%$ of women from non-reminder site completed the OGTT, similar to $14.3 \%$ of women in the RCT non-intervention group). The OGTT screening rates were lower at reminder site A, but $14.4 \%$ of participants from this site did not actually receive the reminder and there was no telephone follow-up. Based on the intention-to-treat model, however, these women were still included in the analyses and participants from this group were still more likely to receive the OGTT.

Our results indicate that there were significantly fewer participants from the non-reminder site completing an OGTT compared to women from the reminder sites, but that there was no differences in the proportion that completed other glucose tests (random glucose test, FPG or HbA1c). Reluctance to perform OGTTs has been demonstrated in the general population of Ontario, ${ }^{17}$ despite it being the best test for screening for diabetes as other types of testing may lead to false-negative results. In a recent large-scale cohort study, women with a history of GDM completed both the OGTT and the FPG; if only the FPG was used, 38\% of those with prediabetes and $75 \%$ of those who met criteria for type 2 diabetes would have been missed. ${ }^{18}$ Similarly, a Canadian study reported that when results from the FPG were used alone, $54 \%$ of women with diabetes would have been identified as normal. ${ }^{9}$ In the current study, only about half of the women who were screened using any glucose test received the recommended test (OGTT, $21.7 \%$ vs. $41.6 \%$ any test), which suggests that the CDA guidelines are not being followed, thus missing opportunities for early intervention. Women with IGT and a history of GDM are more likely to progress to T2DM within 3 years compared to women with IGT and without a history of GDM (38.4\% vs. $25.7 \%$ ). ${ }^{19}$ Many clinical trials have demonstrated that T2DM may be delayed, if not prevented, in these high risk patients through lifestyle modifications and pharmacotherapy. ${ }^{19-22}$ In fact, women with previous GDM may benefit the most from pharmacotherapy..$^{19}$ Continued patient and provider education and service innovations are needed to improve use of an OGTT. 
TABLE 1

Baseline demographic, clinical and metabolic parameters of study participants

\begin{tabular}{|c|c|c|c|c|c|}
\hline & $\begin{array}{c}\text { Non-reminder } \\
\text { group } \\
(\mathrm{N}=117)\end{array}$ & $\begin{array}{l}\text { Reminder } \\
\text { group } A^{\mathrm{a}} \\
(\mathrm{N}=90)\end{array}$ & $\begin{array}{l}\text { Reminder } \\
\text { group } \mathrm{B}^{\mathrm{a}} \\
(\mathrm{N}=55)\end{array}$ & $\begin{array}{l}\text { Excluded } \\
(N=27)\end{array}$ & $p$-value \\
\hline \multicolumn{6}{|l|}{ Baseline characteristics } \\
\hline Maternal age at delivery (years) & $34.0 \pm 5.4$ & $33.5 \pm 5.0$ & $33.3 \pm 4.0$ & $33.7 \pm 5.4$ & .659 \\
\hline Gestational age at GDM diagnosis (weeks) & $26.9 \pm 4.0$ & $27.8 \pm 2.8$ & $27.8 \pm 2.7$ & $26.6 \pm 3.4$ & .106 \\
\hline Pre-pregnancy BMI (kg/m²) & $27.6 \pm 6.8$ & $27.6 \pm 6.0$ & $25.4 \pm 5.3^{b}$ & $28.8 \pm 7.0$ & .039 \\
\hline \multicolumn{6}{|l|}{ Category of BMI (n, \%) } \\
\hline Unknown & $0(0.0)$ & $27(30.0)$ & $1(1.8)$ & & \\
\hline Normal & $53(45.3)$ & $23(25.6)$ & $28(50.9)$ & & \\
\hline Overweight & $28(23.9)$ & $23(25.6)$ & 13 (23.6) & & \\
\hline Obese & $36(30.8)$ & 17 (18.9) & $13(23.6)$ & & \\
\hline Previous GDM & $30(26.3)$ & $20(22.5)$ & $6(11.1)$ & $5(18.5)$ & .082 \\
\hline Primigravida & $35(29.9)$ & $27(30.0)$ & $16(29.1)$ & $5(18.5)$ & .992 \\
\hline Cigarette smoking in pregnancy & $8(6.8)$ & $2(2.6)$ & $5(9.1)$ & $6(22.2)$ & .258 \\
\hline Glucose level in challenge 50-g OGTT $(\mathrm{mmol} / \mathrm{L})$ & $11.1 \pm 2.2$ & $11.8 \pm 1.8$ & $11.2 \pm 1.7$ & $11.5 \pm 1.9$ & .202 \\
\hline Glucose level at $0 \mathrm{~min} 75-\mathrm{g}$ OGTT $(\mathrm{mmol} / \mathrm{L})$ & $5.4 \pm 0.8$ & $5.5 \pm 0.7$ & $5.2 \pm 0.7$ & $5.4 \pm 0.7$ & .35 \\
\hline Glucose level at $60 \mathrm{~min} 75-\mathrm{g}$ OGTT $(\mathrm{mmol} / \mathrm{L})$ & $11.8 \pm 1.3$ & $11.6 \pm 1.4$ & $11.7 \pm 0.9$ & $11.5 \pm 1.1$ & .803 \\
\hline Glucose level at $120 \mathrm{~min} 75-\mathrm{g}$ OGTT $(\mathrm{mmol} / \mathrm{L})$ & $9.4 \pm 1.7$ & $9.6 \pm 1.8$ & $9.6 \pm 1.4$ & $9.3 \pm 1.3$ & .835 \\
\hline \multicolumn{6}{|l|}{ GDM care } \\
\hline GDM treated with insulin & $45(38.5)$ & $25(27.8)$ & $14(25.5)$ & $8(29.6)$ & .131 \\
\hline \multicolumn{6}{|l|}{ Birth outcomes } \\
\hline Gestational age at delivery (weeks) & $38.0 \pm 2.2$ & $38.7 \pm 2.5$ & $39.0 \pm 1.5^{b}$ & $37.3 \pm 3.3$ & .01 \\
\hline Infant birth weight (grams) & $3280.4 \pm 682.6$ & $3408.6 \pm 690.0$ & $3440.7 \pm 513.8$ & $3058.3 \pm 670.0$ & .233 \\
\hline Multiple gestation & $6(5.2)$ & $2(2.8)$ & $0(0.0)$ & $2(7.7)$ & .196 \\
\hline Pregnancy-induced hypertension & $12(10.4)$ & $3(4.2)$ & $5(9.1)$ & $5(19.2)$ & .655 \\
\hline Preeclampsia & $7(6.1)$ & $4(5.6)$ & $2(3.6)$ & $0(0.0)$ & .655 \\
\hline Caesarian section & $50(43.9)$ & 27 (37.5) & $20(36.4)$ & $12(46.2)$ & .552 \\
\hline Family Hx T2DM & $59(50.4)$ & $51(56.6)$ & $27(49.1)$ & $13(48.1)$ & .527 \\
\hline \multicolumn{6}{|l|}{ Income quintile (by postal code) } \\
\hline Missing & $2(1.7)$ & $1(1.1)$ & $0(0.0)$ & $\mathrm{n} / \mathrm{a}$ & $.001^{\mathrm{c}}$ \\
\hline 1 (lowest) & $32(27.4)$ & $32(35.6)$ & $3(5.5)$ & $\mathrm{n} / \mathrm{a}$ & \\
\hline 2 & $19(16.2)$ & $12(13.3)$ & $13(23.6)$ & $\mathrm{n} / \mathrm{a}$ & \\
\hline 3 & $27(23.1)$ & $17(18.9)$ & $13(23.6)$ & $n / a$ & \\
\hline 4 & $19(16.2)$ & $22(24.4)$ & 10 (18.2) & $\mathrm{n} / \mathrm{a}$ & \\
\hline 5 (highest) & 18 (15.4) & $6(6.7)$ & $16(29.1)$ & $\mathrm{n} / \mathrm{a}$ & \\
\hline
\end{tabular}

Abbreviations: BMI, body mass index; GDM, gestational diabetes mellitus; $\mathrm{Hx}$, medical history; $\mathrm{N}$, overall sample size; OGTT, oral glucose tolerance test; p, significance; T2DM, type 2 diabetes mellitus.

Notes: $\mathrm{n} / \mathrm{a}=$ data was not available due to lack of provincial insurance number to link data.

Data represent mean \pm standard deviation or counts (\%).

a Reminder groups A and B were compared to the non-reminder group using chi-square and analysis of variance (ANOVA) analyses.

b Significant versus non-reminder group.

' Reminder group B differs significantly by income quintile compared to reminder group A and the non-reminder group. 
Several factors may play a role in completion of postpartum screening. There are differences in service delivery between our non-reminder and reminder sites. In particular, at the reminder sites all patients were seen by an internist/ endocrinologist, whereas at the non-reminder site all patients saw a high-risk obstetrician and only saw the internist for insulin adjustments. However, given that the rate of screening was the same as in the nonintervention group in our RCT, and the model of care (other than the postpartum reminders) has remained the same at all sites, the difference is most likely due to the implementation of a reminder system. Further, despite our attempts to ensure that the reminder had been received and the patient was available for screening, there were 4 participants who were not sent a reminder and 10 mailed reminders were returned, indicating $9.6 \%$ in the reminder groups who were lost to follow-up. Also, participants in the RCT knew that they were taking part in a research study compared to the current study where women were not aware of this.

Greater contact with health care providers appears to increase screening rates. Visits to health care providers postpartum, either with an endocrinologist or during the 6-week routine follow-up visit where the provider ordered the test, were associated with higher rates of screening. ${ }^{23,24}$ Similarly, in a cohort study of over 14000 women with GDM, visits to an internal medicine or obstetrics/gynecology provider were

TABLE 2

The proportion of study participants from each group who completed glucose screening tests in the first 6 months postpartum

\begin{tabular}{lccc}
\hline & $\begin{array}{c}\text { Non-reminder } \\
\text { group } \\
(\mathbf{N}=117)\end{array}$ & $\begin{array}{l}\text { Reminder } \\
\text { group A } \\
(\mathbf{N}=90)\end{array}$ & $\begin{array}{c}\text { Reminder } \\
\text { group B } \\
(\mathbf{N}=55)\end{array}$ \\
\hline OGTT & $16(13.7 \%)$ & $21(23.3 \%)$ & $20(36.4 \%)^{\mathrm{a}}$ \\
Random/fasting glucose & $31(26.5 \%)$ & $23(25.6 \%)$ & $12(21.8 \%)$ \\
HbA1c & $16(13.7 \%)$ & $12(13.3 \%)$ & $9(16.4 \%)$ \\
Any glucose test & $44(37.6 \%)$ & $38(42.2 \%)$ & $27(49.1 \%)$ \\
\hline
\end{tabular}

Abbreviations: HbA1c, glycated hemoglobin test; N, sample size; OGTT, oral glucose tolerance test; $p$, statistical significance.

${ }^{\text {a }} p=.01$, reminder group B vs. non-reminder group. independent and significant predictors of postpartum screening in the year postdelivery. ${ }^{18}$ In our practice, no routine postpartum appointments are made with the internist/endocrinologist. Our study was limited in that we were not able to access the out-of-hospital records for postpartum care by an obstetrician or primary care provider.

Although there are no other direct studies comparing models of care delivery, case management may improve screening rates. One prospective cohort study followed women with GDM who were provided laboratory requisitions upon hospital discharge and also contacted at home by a casemanager who could perform the test; this led to an OGTT screening rate of $41 \% .{ }^{25}$ At Kaiser Permanente in Northern California, screening rates utilizing an OGTT increased from $16.6 \%$ to $71.5 \%$ when a nursemanaged care program was instituted. ${ }^{18}$

Women at highest risk of developing T2DM may not be returning for screening. In our study, women on insulin were more likely to complete postpartum screening, but obese women were least likely to do so, with a rate of only $28.8 \%$ completing any glucose screening. One prospective cohort study followed women with GDM who were provided laboratory requisitions upon hospital discharge and also contacted at home by a case-manager who could perform the test. ${ }^{25}$ The women who did not return had a greater incidence of previous GDM, higher diagnostic glucose levels

and were more likely to have been taking insulin during pregnancy, suggesting that women with less severe GDM were more likely to return for follow-up. We need further research on perceptions of risk visà-vis screening to find out why high-risk women are not being screened.

Our study has several limitations. We only followed women for 6 months postpartum in keeping with the CDA screening recommendations. (In our RCT study, we included testing done within one year.) The results from other studies are varied: one found that women will delay their testing up to 428 days postpartum, ${ }^{14}$ whereas another demonstrated that $94.3 \%$ of women completed it by 12 weeks postpartum. ${ }^{25}$ However, the performance of this study in one urban multicultural centre may limit the generalizability of the results.

In summary, the current study shows that reminders are an effective method of reinforcing guidelines for postpartum diabetes screening. However, the majority of women continue to not receive any glucose screening, let alone the recommended OGTT. Care providers should consider implementing a structured approach to postpartum followup of women with a history of GDM. Further studies should assess different methods of postpartum reminders and barriers to implementation.

\section{Acknowledgements}

We would like to thank Sharon Rouatt, Diabetes Educator; Pauline BrazeauGravelle, Clinical Dietician; Filamena DeSousa, Diabetes Educator; and Anjie Huang, Statistician. Funding for Alison Shea was provided by the Canadian Institutes of Health Research (CIHR) studentship. Funding for the study was provided by the Obstetrics Maternal \& Newborn Investigation (OMNI) research group, The Ottawa Hospital. Dr. Baiju Shah receives salary support from the Canadian Institutes of Health Research, the Canadian Diabetes Association, and the Banting and Best Diabetes Centre of the University of Toronto. No authors have a conflict of interest with this study. 
TABLE 3

Adjusted logistic regression models for predicting postpartum glucose screening among women with a history of GDM

\begin{tabular}{|c|c|c|c|c|}
\hline Outcome & Effect & Adjusted OR & $95 \% \mathrm{Cl}$ & $p$-value \\
\hline \multicolumn{5}{|l|}{ OGTT } \\
\hline & Site & & & .029 \\
\hline & Reminder A vs. non-reminder & 1.57 & $0.66-3.70$ & \\
\hline & Reminder B vs. non-reminder & 3.10 & $1.35-7.14$ & \\
\hline & Age & & & .262 \\
\hline & $<30$ vs. $\geq 40$ years & 2.51 & $0.58-10.83$ & \\
\hline & $30-39$ vs. $\geq 40$ years & 3.06 & $0.79-11.84$ & \\
\hline & Prior GDM & 0.49 & $0.20-1.23$ & .131 \\
\hline & BMI & & & .134 \\
\hline & Normal vs. obese & 2.42 & $0.92-6.36$ & \\
\hline & Overweight vs. obese & 3.30 & $1.20-9.06$ & \\
\hline \multicolumn{5}{|c|}{ GDM treatment } \\
\hline & Diet vs. insulin & 0.38 & $0.18-0.80$ & .012 \\
\hline & Family Hx T2DM & 1.07 & $0.55-2.05$ & .845 \\
\hline & SES quintiles & & & .635 \\
\hline & 1 (lowest) vs. 5 (highest) & 0.89 & $0.29-2.63$ & \\
\hline & 2 vs. 5 & 0.96 & $0.33-2.84$ & \\
\hline & 3 vs. 5 & 0.85 & $0.29-2.47$ & \\
\hline & 4 vs. 5 & 1.68 & $0.59-4.77$ & \\
\hline \multicolumn{5}{|l|}{ Any test } \\
\hline & Site & & & .734 \\
\hline & Reminder A vs. non-reminder & 1.09 & $0.56-2.13$ & \\
\hline & Reminder B vs. non-reminder & 1.33 & $0.65-2.71$ & \\
\hline & Age & & & .595 \\
\hline & $<30$ vs. $\geq 40$ years & 0.74 & $0.28-1.98$ & \\
\hline & $30-39$ vs. $\geq 40$ years & 1.05 & $0.44-2.49$ & \\
\hline & Prior GDM & 0.68 & $0.35-1.34$ & .264 \\
\hline & BMI & & & .032 \\
\hline & Normal vs. obese & 2.40 & $1.16-5.01$ & \\
\hline & Overweight vs. obese & 3.10 & $1.42-6.77$ & \\
\hline \multicolumn{5}{|c|}{ GDM treatment } \\
\hline & Diet vs. insulin & 0.60 & $0.32-1.12$ & .107 \\
\hline & Family Hx T2DM & 0.77 & $0.45-1.31$ & .328 \\
\hline & SES quintiles & & & .195 \\
\hline & 1 (lowest) vs. 5 (highest) & 0.92 & $0.38-2.21$ & \\
\hline & 2 vs. highest & 1.33 & $0.54-3.31$ & \\
\hline & 3 vs. highest & 0.85 & $0.35-2.04$ & \\
\hline & 4 vs. highest & 2.09 & $0.85-5.13$ & \\
\hline
\end{tabular}

Abbreviations: BMI, body mass index; $\mathrm{Cl}$, confidence interval; GDM, gestational diabetes mellitus; Hx, medical history; OGTT, oral glucose tolerance test; OR, odds ratio; SES, socioeconomic status; T2DM, type 2 diabetes mellitus.

Notes: The category listed first on each line represents the reference group. 


\section{References}

1. Dyck R, Klomp H, Tan LK, Turnell RW, Boctor MA. A comparison of rates, risk factors, and outcomes of gestational diabetes between aboriginal and non-aboriginal women in the Saskatoon health district. Diabetes Care. 2002;25:487-93.

2. Rodrigues S, Robinson E, Gray-Donald K. Prevalence of gestational diabetes mellitus among James Bay Cree women in northern Quebec. CMAJ. 1999;160:1293-97.

3. Feig DS, Zinman B, Wang X, Hux JE. Risk of development of diabetes mellitus after diagnosis of gestational diabetes. CMAJ. 2008;179:229-34.

4. Kim C, Newton KM, Knopp RH. Gestational diabetes and the incidence of type 2 diabetes: a systematic review. Diabetes Care. 2002;25:1862-68.

5. Retnakaran R, Qi Y, Sermer M, Connelly PW, Hanley AJ, Zinman B. Glucose intolerance in pregnancy and future risk of pre-diabetes or diabetes. Diabetes Care. 2008;31:2026-31.

6. Bellamy L, Casas JP, Hingorani AD, Williams D. Type 2 diabetes mellitus after gestational diabetes: a systematic review and meta-analysis. Lancet. 2009;373:1773-9.

7. Canadian Diabetes Association Clinical Practice Guidelines Expert Committee. Canadian Diabetes Association 2008 clinical practice guidelines for the prevention and management of diabetes in Canada. Can J Diabetes. 2008;32 Suppl 1:S1-S201.

8. Metzger BE, Buchanan TA, Coustan DR, de Leiva A, Dunger DB, Hadden DR, Hod M, Kitzmiller JL, Kjos SL, Oats JN, Pettitt DJ, Sacks DA, Zoupas C. Summary and recommendations of the Fifth International Workshop-Conference on Gestational Diabetes Mellitus. Diabetes Care. 2007;30 Suppl 2:S251-60.

9. Reinblatt SL, Morin L, Meltzer SJ. The importance of a postpartum $75 \mathrm{~g}$ oral glucose tolerance test in women with gestational diabetes. J Obstet Gynaecol Can. 2006;28:690-4.
10. Ogonowski J, Miazgowski T. The prevalence of 6 weeks postpartum abnormal glucose tolerance in Caucasian women with gestational diabetes. Diabetes Res Clin Pract. 2009;84:239-44.

11. Retnakaran R, Qi Y, Sermer M, Connelly PW, Zinman B, Hanley AJ. Comparison of National Diabetes Data Group and American Diabetes Association diagnostic criteria for gestational diabetes in their identification of postpartum risk of glucose intolerance. Diabetes Res Clin Pract. 2009;85:40-6.

12. Clark HD, van Walraven C, Code C, Karovitch A, Keely E. Did publication of a clinical practice guideline recommendation to screen for type 2 diabetes in women with gestational diabetes change practice? Diabetes Care. 2003;26:265-8.

13. Dietz PM, Vesco KK, Callaghan WM, Bachman DJ, Bruce FC, Berg CJ, England LJ, Hornbrook MC. Postpartum screening for diabetes after a gestational diabetes mellitus-affected pregnancy. Obstet Gynecol. 2008;112:868-74.

14. Smirnakis KV, Chasan-Taber L, Wolf M, Markenson G, Ecker JL, Thadhani R. Postpartum diabetes screening in women with a history of gestational diabetes. Obstet Gynecol. 2005;106:1297-303.

15. Bentley-Lewis R, Levkoff S, Stuebe A, Seely EW. Gestational diabetes mellitus: postpartum opportunities for the diagnosis and prevention of type 2 diabetes mellitus. Nat Clin Pract Endocrinol Metab. 2008;4:552-8.

16. Clark HD, Graham ID, Karovitch A, Keely EJ. Do postal reminders increase postpartum screening of diabetes mellitus in women with gestational diabetes mellitus? A randomized controlled trial. Am J Obstet Gynecol. 2009;200:634.e1-7.

17. Wilson SE, Lipscombe LL, Rosella LC, Manuel DG. Trends in laboratory testing for diabetes in Ontario, Canada 1995-2005: a population-based study. BMC Health Serv Res. 2009;9:41.
18. Ferrara A, Peng T, Kim C. Trends in postpartum diabetes screening and subsequent diabetes and impaired fasting glucose among women with histories of gestational diabetes mellitus: a report from the Translating Research Into Action for Diabetes (TRIAD) Study. Diabetes Care. 2009;32:269-74.

19. Ratner RE, Christophi CA, Metzger BE, Dabelea D, Bennett PH, Pi-Sunyer X, Fowler S, Kahn SE; Diabetes Prevention Program Research Group. Prevention of diabetes in women with a history of gestational diabetes: effects of metformin and lifestyle interventions. J Clin Endocrinol Metab. 2008;93:4774-9.

20. Ratner RE. Prevention of type 2 diabetes in women with previous gestational diabetes. Diabetes Care. 2007;30:\$242-5.

21. Xiang AH, Peters RK, Kjos SL, Marroquin A, Goico J, Ochoa C, Kawakubo M, Buchanan TA. Effect of pioglitazone on pancreatic beta-cell function and diabetes risk in Hispanic women with prior gestational diabetes. Diabetes. 2006;55:517-22.

22. Buchanan TA, Xiang AH, Peters RK, Kjos SL, Marroquin A, Goico J, Ochoa C, Tan S, Berkowitz K, Hodis HN, Azen SP. Preservation of pancreatic beta-cell function and prevention of type 2 diabetes by pharmacological treatment of insulin resistance in high-risk Hispanic women. Diabetes. 2002;51:2796-803.

23. Kim C, Tabaei BP, Burke R, McEwen LN, Lash RW, Johnson SL, Schwartz KL, Bernstein SJ, Herman WH. Missed opportunities for type 2 diabetes mellitus screening among women with a history of gestational diabetes mellitus. Am J Public Health. 2006;96:1643-8.

24. Greenberg LR, Moore TR, Murphy H. Gestational diabetes mellitus: antenatal variables as predictors of postpartum glucose intolerance. Obstet Gynecol. 1995;86:97-101.

25. Hunt KJ, Conway DL. Who returns for postpartum glucose screening following gestational diabetes mellitus? Am J Obstet Gynecol. 2008;198:404.e1-6. 